\title{
Telefonaktion am 20. Juli 2008
}

\author{
Renate Maltry \\ Vizepräsidentin des djb, Rechtsanwältin, \\ Fachanwältin Erbrecht, Fachanwältin Familien- \\ recht, München
}

Die Regionalgruppe Münster hat es schon mehrfach vorgemacht. Im Rahmen der Münsteraner Frauenaktionswochen „Frauen im Recht“ bot sie zuletzt Ende 2005 in Zusammenarbeit mit der Arbeitsgemeinschaft Anwältinnen im DAV eine Anwältinnenhotline an. Anwältinnen beantworteten kostenlos und unverbindlich allgemeine Rechtsfra-

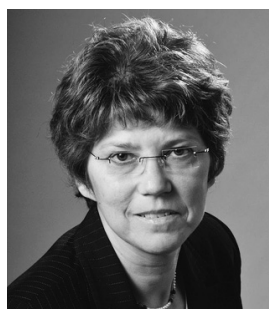

Petra Hecker-
Buschkamp
Rechtsanwältin und
Fachanwältin für
Familienrecht, Bielefeld

gen. Die Informationen zur Hotline und die Telefonnummern, die die Ratsuchenden anrufen sollten, verbreitete die Tagespresse. Der Berliner Tagesspiegel und andere lokale Zeitungen bieten ebenfalls regelmäßig Telefonaktionen an, auch unter Mitarbeit von Rechtsanwältinnen aus dem djb.

Der Bundesvorstand hatte Ende letzten Jahres beschlossen, in Zusammenarbeit mit überregionalen (Tages-)Zeitungen bundesweite Telefonaktionen speziell für Rechtsanwältinnen im djb auf die Beine zu stellen. Beabsichtigt war, Anwältinnen in der Öffentlichkeit präsenter und die vielen kompetenten Rechtsanwältinnen im djb sichtbarer zu machen. Im djb-Newsletter für Mitglieder vom Dezember 2007 wurde bei den Anwältinnen das Interesse an einer

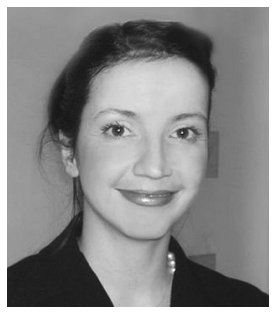

Dr. Vanessa Hohenbleicher

Rechtsanwältin und Fachanwältin für Familienrecht, München

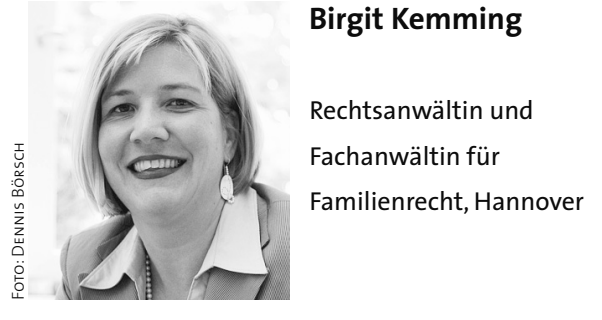

solchen Aktion und potentielle Rechtsgebiete abgefragt.

Jutta Wagner, Präsidentin des djb, führte unter anderem - nach Vermittlung unserer früheren 1. Vorsitzenden Rechtsanwältin Renate Damm - entsprechende Gespräche mit der Bild am Sonntag. Jetzt, anlässlich der ersten BGH-Entscheidung vom 16. Juli 2008 zum Bedarf und zur Dauer des Betreuungsunterhalts der Mutter eines nichtehelichen Kindes, fand mit der Bild am Sonntag vom 20. Juli 2008 die erste bundesweite Telefonaktion statt. Die Unterhaltsrechtsreform war am 1. Januar 2008 in Kraft getreten. Weil der zum 1. Januar 2008 geänderte Anspruch auf Betreuungsunterhalt der Mutter eines nichtehelichen Kindes ( $\int 1615$ l Abs. 2 BGB) und der Anspruch auf nachehelichen Betreuungsunterhalt

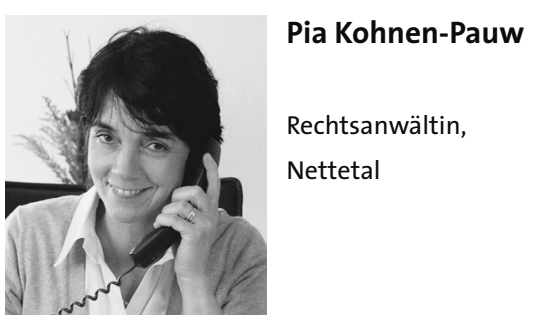

( $\int 1570$ BGB) durch das Unterhaltsrechtsänderungsgesetz einander weitgehend angeglichen worden sind, hat diese BGH-Entscheidung auch erhebliche Auswirkungen auf die Dauer des nachehelichen Betreuungsunterhalts.

Sieben Rechtsanwältinnen - Agnes Schmidt, Petra Hecker-Buschkamp, Dr. Vanessa Hohenbleicher, Birgit Kemming, Pia Kohnen-Pauw, Anne Lütkes und Christine Schomerus - haben als Mitglieder des djb an der Telefonaktion zum Unterhaltsrecht teilgenommen. Alle Anwältinnen wurden in der Ausgabe der entsprechenden Bild-Ausgabe namentlich mit Foto und Hinweis auf die djbMitgliedschaft präsentiert. Es war ein voller Erfolg. Mit 6.775 Anrufen von 10.00 bis 14.00 Uhr waren die Anwältinnen beschäftigt. Der Beratungsbedarf, der alle gesellschaftlichen Schichten betraf, war, wie sich zeigte, enorm groß. Pro Anwältin konnten maximal 48 Anrufe bearbeitet werden, was ohnehin

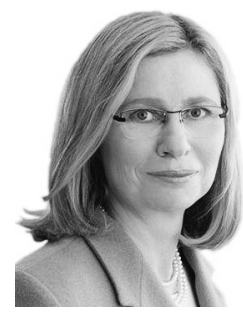

\section{Anne Lütkes}

Rechtsanwältin und

Fachanwältin für

Familienrecht, Köln

schon eine Höchstleistung war. Alle Anwältinnen berichteten, dass es ihnen trotz des enormen Andranges Spaß gemacht habe.

In der Bild am Sonntag vom 27. Juli 2008 wurden auf zwei Seiten die von den Anwältinnen eingereichten wesentlichen Fragen mit Antworten und einem Foto jeder Anwältin veröffentlicht. Die Zeitung hat angekündigt, weitere Telefonaktionen mit den Anwältinnen des djb durchführen zu wollen. Dies kann in jedem interessanten Themenbereich erfolgen. Maßgeblich ist dabei, dass eine wichtige höchstrichterliche Entscheidung oder eine Gesetzesänderung vorliegen, die Beratungsbedarf nach sich ziehen.

Wenn Sie Interesse an einer Teilnahme haben, können Sie sich - sofern noch nicht geschehen - über das im Mitgliederbereich unserer Homepage abrufbare Formular anmelden. Als potentielle

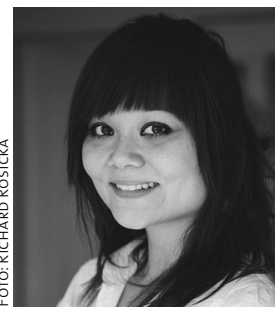

Agnes Schmidt

Rechtsanwältin, Groß-Gerau 


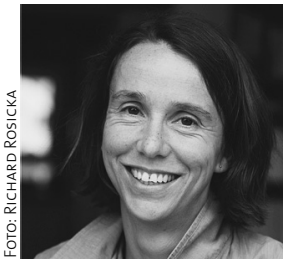

Christine Schomerus

Rechtsanwältin,

Dossenheim

Fachgebiete stehen derzeit folgende $\mathrm{Ru}$ briken zur Verfügung:
- Unterhaltsrecht

- Familienrecht allgemein

- Arbeitsrecht

- Strafrecht (Nebenklage)

- Erbrecht

- Betreuungsrecht/Vollmachten

- Ausländerrecht

- Sozialrecht (Hartz-IV)

- Mietrecht
Wenn eine weitere Telefonaktion in $\mathrm{Zu}$ sammenarbeit mit einer Zeitung ansteht - was meist sehr kurzfristig entschieden wird - meldet sich die Geschäftsstelle bei allen für das betreffende Fachgebiet eingetragenen Kolleginnen per E-Mail. Die Möglichkeit der Teilnahme richtet sich dann auch nach dem Eingang Ihrer Rückmeldung.

\section{Der djb gratuliert}

\section{Ingrid Baer, Direktorin des Internationalen Sozialdienstes i. R. zu ihrem 70. Geburtstag}

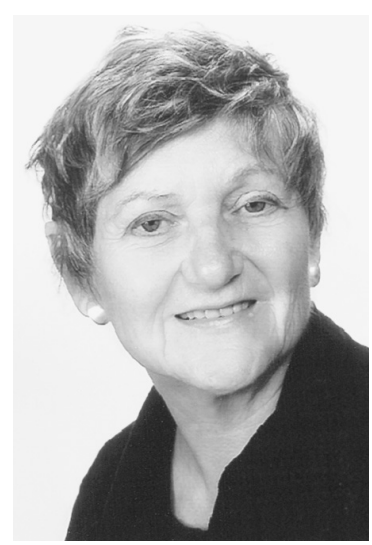

Ingrid Baer wurde 1938 in Kusel/ Pfalz geboren und machte dort mit 17 Jahren das Abitur. Außer dem deutschen Gymnasium besuchte sie ein englisches und ein französisches Internat. Das juristische Referendarexamen legte sie 1960 in Saarbrücken ab, das 2. Examen 1964 in Neustadt/ Weinstraße. Inzwischen hatte sie geheiratet und gebar 1964 ihren ersten Sohn. Der zweite Sohn folgte 1967.

Trotz zweier Prädikatsexamen war der Einstieg ins Berufsleben schwierig, die Vorbehalte gegenüber einer Mutter von zwei kleinen Kindern waren groß. Ingrid Baer begann mit einer Teilzeitarbeit in einem Industrieunternehmen in Köln und baute dort die Abteilung für Warenzeichen- und Wettbewerbsrecht auf. Ihre Interessen waren aber immer der soziale Bereich und das Familienrecht. 1970 begann sie beim Landschaftsverband Rheinland und nach sechsmonatiger Einarbeitung wurde ihr eine verantwortungsvolle Position im Fachbereich Jugendhilfe angeboten.

Nachdem ihr Mann aus beruflichen Gründen nach Frankfurt wechselte, musste sie sich neu orientieren und begann dort als Referentin beim Deutschen Verein für öffentliche und private Fürsorge. 1972 wurde ihr die Leitung des Internationalen Sozialdienstes angeboten, das hieß Vollzeittätigkeit mit zwei kleinen Kindern. Es war jedoch eine einmalige Chance. Ihr Mann redete ihr zu und versprach häusliche Unterstützung.

Beim Internationalen Sozialdienst erwartete sie ein großes neues Aufgabengebiet, vorderhand die Verwaltung und Organisation der deutschen Zweigstelle sowie der nationalen und internationalen Zusammenarbeit (weltweit cirka 100 Fachstellen des Gesamtverbandes International Social Service).

Ingrid Baers primäres Interesse galt jedoch der fachlichen Arbeit: Zunächst stand die große Reform des Adoptionsvermitt- lungsrechts im Vordergrund. Möglichst viele Heimkinder, auch ältere und behinderte Kinder, sollten eine Familie bekommen. Hinzu kam die Vermittlung von verlassenen Kindern aus der so genannten 3. Welt in deutsche Familien. Internationale Empfehlungen zum Verfahren wurden entwickelt. Sodann galt die Sorge den Dauerpflegekindern, sie sollten ein Recht auf Verbleib in ihrer „sozialen Familie“ erhalten. Es folgte die Unterstützung bei der Suche nach ihren Wurzeln für Menschen, die ohne leibliche Angehörige aufgewachsen waren. Die Aufnahme minderjähriger Flüchtlinge aus Vietnam führte 1980 zur Entwicklung eines bundesweiten Beratungsprogramms bezüglich der Betreuung und Eingliederung dieser Jugendlichen.

Ingrid Baers Arbeit in der Familienrechtskommission des Europarates, mit der Möglichkeit zur Rechtsvergleichung moderner Entwicklungen, führte zu Vorschlägen zur Neugestaltung im Sorge- und Umgangsrecht: gemeinsames elterliches Sorgerecht nach Scheidung und eine neue Sicht auf die Beziehung zwischen Kind und nichtehelichem Vater mit den notwendigen rechtlichen Konsequenzen. Die Prävention von internationalen Kindesentziehungen durch Mitwirkung an der Entwicklung internationaler Konventionen der Haager Konferenz für Internationales Privatrecht war eine weitere Aufgabe. Ingrid Baer hat durch Mitarbeit in Fachausschüssen und Konferenzen auf nationaler und internationaler Ebene, insbesondere aber durch zahlreiche Publikationen in der Fachpresse dazu beitragen können, dass Regelungen und Verfahrensweisen entwickelt wurden, die Kinder schützen und ihre eigenen Rechte sichern, eine dankbare Aufgabe, die ihr viel Freude gemacht hat.

Dem djb trat Ingrid Baer schon als Studentin bei. Sie hielt Vorträge bei djb-Kongressen und Arbeitstagungen und vor allem war sie ein langjähriges aktives Mitglied der Kommission Familienrecht (später: Zivilrecht). Aktuell arbeitet sie in der Kommission Kinderschutz mit. Die Arbeit im djb war ihr immer sehr wichtig.

(Dr. Angelika Nake)

Ingrid Baers Anschrift ist: Schillerstraße 11a, 61440 Oberursel. 